\title{
Les leçons de républicanisme de Thomas Paine
} (1802-1807)

Thomas Paine's Lessons on Republicanism (1802-1807)

\section{Marc Belissa}

Traducteur : Stephen Clay et Allan Potofsky

\section{(2) OpenEdition}

\section{Édition électronique}

URL : https://journals.openedition.org/ahrf/11937

DOI : 10.4000/ahrf.11937

ISSN : 1952-403X

Éditeur :

Armand Colin, Société des études robespierristes

\section{Édition imprimée}

Date de publication : 1 mars 2011

Pagination : 59-84

ISBN : 978-2-200-92677-9

ISSN : 0003-4436

Référence électronique

Marc Belissa, "Les leçons de républicanisme de Thomas Paine (1802-1807) », Annales historiques de la Révolution française [En ligne], 363 | janvier-mars 2011, mis en ligne le 01 mars 2014, consulté le 23 avril 2022. URL : http://journals.openedition.org/ahrf/11937 ; DOI : https://doi.org/10.4000/ahrf.11937 


\title{
LES LEÇONS DE RÉPUBLICANISME DE THOMAS PAINE (1802-1807)
}

Marc BELISSA

\begin{abstract}
Les écrits politiques des dernières années de Thomas Paine (1802-1807) ont été largement négligés par l'historiographie. Ils posent pourtant des questions fondamentales pour l'évolution du républicanisme en Amérique. Trois thèmes émergent de ce corpus : tout d'abord, celui des factions et des partis dans la république, deuxièmement, la question de la perfectibilité du régime républicain et des mécanismes constitutionnels qui doivent en assurer l'expression, et enfin la question des formes que doit prendre la « mémoire » du moment fondateur révolutionnaire dans le maintien de la vertu républicaine. Ces trois thèmes dessinent ce qui me semble être des «leçons de républicanisme» à destination de la génération nouvelle.
\end{abstract}

Mots-clés: Thomas Paine, républicanisme, factions, fédéralistes, partis, Révolution américaine.

Dans une recherche récente consacrée à la « légende grise » des dernières années de la vie de Thomas Paine en Amérique ${ }^{1}$, j'ai été frappé de voir à quel point sa production politique des années 1802-1807 était à la fois importante (quantitativement et qualitativement), mais surtout totalement négligée par l'historiographie. Ce sont ces écrits que j'entends analyser ici.

Dans l'historiographie ancienne et récente, la période finale de la vie de Thomas Paine disparaît en effet le plus souvent ou n'est traitée

(1) Marc Belissa, « La légende grise des dernières années de Thomas Paine en Amérique, 1802-1809 », AHRF, 2010-2, p. 133-172. 
que comme un appendice à la carrière du vieux révolutionnaire. Ainsi, à part les biographies qui l'abordent en épilogue, elle n'a suscité aucune recherche même si un ouvrage de Nathalie Caron étudie la question religieuse dans les années 1790-1800'. Seul un article de Jerry Knudson traite du moment du retour de Paine en Amérique, mais ne va pas audelà de l'année $1803^{3}$. Il faut également citer un texte de Bernard Vincent consacré au rôle de Paine dans la question de la Louisiane ${ }^{4}$. Mais aucune étude ne s'est intéressée en tant que telle à la production de Paine des années 1802-1807 dans le domaine politique. Aucun article n'analyse notamment les huit Letters to the Citizens of the United States publiées entre 1802 et 1805 ou ses textes consacrés à la question des réformes constitutionnelles en Pennsylvanie ou encore ses articles dédiés à la défense de Jefferson contre les Fédéralistes ${ }^{5}$.

Dans cette étude, je me concentrerai sur les textes de Paine consacrés aux questions du républicanisme aux États-Unis, en laissant de côté les textes sur l'intégration de la Louisiane ${ }^{6}$. Ainsi limité, le corpus sur lequel je m'appuie comprend, outre la correspondance avec Jefferson, Madison, etc., les huit Letters to the Citizens of the United States publiées entre le 15 novembre 1802 et le 7 juin 1805, les articles isolés publiés dans la presse contre la faction fédéraliste, les papiers consacrés au mouvement de réforme constitutionnelle en Pennsylvanie et à New York ${ }^{7}$.

(2) Nathalie CARon, Thomas Paine contre l'imposture des prêtres, Paris, L'Harmattan, 1999.

(3) Jerry Knudson, « The Rage over Tom Paine. Newspaper Reaction to his Homecoming in $1802 »$, New York Historical Society Quarterly, vol. 53, 1969, p. 34-63.

(4) Dans le recueil The Transatlantic republican, Thomas Paine and the Age of Revolutions, New York, Rodopi, 2005.

(5) Pour un florilège d'appréciations négatives de l'historiographie sur les derniers textes de Paine, voir notamment Jerry Knudson, op. cit., p. 55. Cecelia Kenyon, "Where Paine Went Wrong », American Political Science Review, vol. 45, n 4, déc. 1951, p. 1086-1099. Jules Ayer, Thomas Paine, New York, Atheneum, 1988, p. 165. Jack Fruchtman, Thomas Paine, Apostle of Freedom, New York, 1994, p. 396-397. Craig Nelson, Thomas Paine, Enlightenment, Revolution, and the Birth of Modern Nations, Penguin Books, 2007, p. 308.

(6) J'y ai consacré un court article paru en ligne sur revolution-française.net en janvier 2010. «Thomas Paine et la fondation républicaine en Louisiane », http://revolution-francaise. net/2010/01/01/354-thomas-paine-fondation-republicaine-louisiane. Les articles sur les questions religieuses et théologiques publiés dans The Prospect, les textes consacrés à la situation en Europe ne seront pas pris en compte, bien qu'évidemment, l'analyse de la situation européenne a des implications sur les textes « américains » de Paine. De même, les questions religieuses sont aussi politiques (Nathalie Caron l'a abondamment montré).

(7) Les éditions utilisées ici sont: The Writings of Thomas Paine, édition Moncure Daniel Conway, 4 vol., New York et Londres, 1893-1896, tomes III et IV. The Complete Writings of Thomas Paine, collected and edited by Philip S. Foner, New York, Citadel Press, 1969 [c1945], 2 vols. Collected Writings, édition Eric Foner, Library of America, 1976. Toutes les citations historiographiques et celles des œuvres de Paine ci-dessous sont traduites par l'auteur. 
Trois thèmes émergent de ce corpus : tout d'abord, celui des factions et des partis dans la république, deuxièmement, la question de la perfectibilité du régime républicain et des mécanismes constitutionnels qui doivent en assurer l'expression, et enfin la question des formes que doit prendre la « mémoire » du moment fondateur révolutionnaire dans le maintien de la vertu républicaine. Ces trois thèmes dessinent ce qui me semble être des « leçons de républicanisme » à destination de la génération nouvelle.

\section{Contre la «faction » fédéraliste, pour la « révolution de 1800 »}

Les textes de Paine des dernières années s'inscrivent dans ce que les républicains appellent alors la « révolution de $1800 »$. En effet, la défaite des Fédéralistes et l'élection de Jefferson inaugurent une nouvelle période de l'histoire des États-Unis. Le projet fédéraliste et hamiltonien de construction d'une puissance sur le modèle anglais est battu, c'est désormais celui d'un développement de l'Amérique vers l'intérieur qui s'impose. Par ailleurs, la « révolution de 1800 » n'est pas un simple changement de parti au pouvoir mais un tournant démocratique. La victoire républicaine affaiblit l'élitisme et encourage une pratique politique élargie ${ }^{8}$.

Dès son adresse inaugurale, Jefferson réaffirme les principes républicains. Le gouvernement doit être « sage et frugal ». Les droits d'expression de la minorité fédéraliste seront respectés mais les attentes de la majorité républicaine aussi. Pour Jefferson, sa présidence doit d'abord revenir au véritable esprit républicain, celui de 1776. La victoire de 1801 est donc d'abord une « restauration» du républicanisme des origines. Il faut d'abord inverser l'évolution centralisatrice impulsée par Hamilton, mettre fin au processus de constitution du système fisco-militaro-financier voulu par les Fédéralistes et ouvrir largement la démocratie américaine en regardant vers l'Ouest. Jefferson entend également renverser l'évolution hamiltonienne vers un renforcement militaire des États-Unis : le pouvoir civil doit se subordonner l'armée et encourager la constitution de milices pour éviter une armée fédérale professionnelle dangereuse pour la liberté. Jefferson entend rembourser au plus vite la dette extérieure et intérieure, faire des économies et défendre les droits des citoyens contre l'État fédéral. Enfin, Jefferson souhaite favoriser une liberté totale de religion, d'expression et de presse.

(8) Sur la « Révolution de 1800 », voir Henry AdAms, History of the US during the Administration of Thomas Jefferson and James Madison, 1889-1890, 9 vol; Dan A. Sisson, The American Revolution of 1800, New York, A. Knopf, 1974; Drew Mc CoY, The Elusive Republic: Political Economy in Jeffersonian America, Chapel Hill, University of North Carolina Press, 1980. 
Comme tous les républicains, Paine considère que la période dite « fédéraliste » de 1789 à 1800 est marquée par l'apparition d'une " faction » dont les principaux dirigeants sont Hamilton, Adams et Gouverneur Morris (Washington étant considéré plutôt comme la dupe de ladite «faction»). Cette faction défend une ligne qui se révèle peu à peu dans les débats à partir de 1790-1791 et apparaît au grand jour à partir de 1794-1795 lors de la signature du traité Jay puis tente de renverser les principes fondateurs de la république lors de la période de la Quasi-War avec la France. Si les Fédéralistes appliquent une stratégie politique, Paine considère que ce qui caractérise cette faction, comme toutes les factions, est l'absence de principes, ou comme il l'écrit la « nullité de principes ». Pour Paine, la ligne des Fédéralistes n'est pas fondée sur des principes «républicains », mais sur une conception « monarchique » au sens philosophique du terme (qui n'est pas forcément liée à la présence d'un roi) et donc non-fondée sur les principes, puisque, selon Paine, « la monarchie n'en a pas ». Il n'y a donc pas de contradiction pour Paine lorsqu'il proclame l'absence de principes des Fédéralistes tout en décrivant les objectifs politiques qu'ils entendent atteindre et la stratégie qu'ils suivent. Là où bon nombre de biographes n'ont vu que des vitupérations et des accusations personnelles sans fondement, il me semble qu'il faut plutôt voir dans ces textes de Paine une conception républicaine de ce que sont « les principes » par opposition à ce qu'est une « faction », c'est-à-dire un groupe sans principes républicains, mais doté d'une ligne politique visant à faire rétrograder la Révolution américaine vers un régime « monarchique » ou " monocratique ». Plus généralement, Paine en arrive à une sorte de théorie de l'apparition des factions dans une république vertueuse et à leur échec inévitable pour peu qu'existe une résistance des citoyens et que subsiste la mémoire des principes fondateurs de la République. Telle est la première des « leçons de républicanisme » de Paine.

Quelles sont les caractéristiques des factions en général et de la faction "fédéraliste» en particulier? Paine l'explique tout au long de ses huit Letters to the Citizens of the United States 9 . Dès la première du 15 novembre 1802, il résume son propos :

(9) Les huit Letters to the Citizens of the United States And particularly to the Leaders of the Federal Faction sont publiées entre le 15 novembre 1802 (donc deux semaines après son retour aux États-Unis) et le 7 juin 1805. Les cinq premières (15, 19, 26 novembre, 3 décembre 1802, 2 février 1803) sont publiées dans un des principaux journaux républicains, le National Intelligencer, la sixième dans l'Aurora de Philadelphie le 12 mars 1803, la septième le 21 avril dans le True American de Trenton, et enfin la dernière paraît le 7 juin 1805 dans le National Intelligencer. Les citations suivantes des Letters 1 à 7 sont toutes tirées du tome III de l'édition Conway, celles de la Letter VIII du tome II de l'édition de Philip S. FonER. 
«Une faction, agissant dans l'ombre, apparaissait en Amérique; ses membres avaient perdu de vue les principes premiers. Ils commençaient à considérer le gouvernement comme un monopole fructueux, et le peuple comme une propriété héréditaire ${ }^{10}$.

La première caractéristique est donc la dissimulation. Une faction ne peut agir ouvertement, elle est nécessairement secrète et cache ses objectifs en en proclamant d'autres ou en instrumentalisant les passions et/ou les opinions des citoyens pour les faire dévier des principes ${ }^{11}$.

Deuxième caractéristique : les membres de la faction ont perdu de vue ou ont renoncé aux principes de la République pour défendre des intérêts particuliers ou pire encore, n'ont jamais eu le moindre principe républicain. La cible favorite de Paine est celui qui apparaissait comme le «théoricien » de la faction, John Adams, l'auteur des Thoughts on Government (1776), de la Defence of the Constitutions of the United States (1787) et des Discourses on Davila (1790). Adams incarnait pour Paine le défenseur par excellence de la monarchie à l'anglaise, sa définition de la République n'étant appuyée sur aucun principe : «C'est, ditil, l'empire des lois et non des hommes. Mais comme les lois peuvent être aussi bien mauvaises que bonnes, un empire des lois peut être le meilleur des gouvernements ou la pire des tyrannies $\gg^{12}$. Pour Paine, la république n'est pas seulement ce que nous appellerions aujourd'hui un «État de droit ». Un État " réglé » n'est pas une véritable république sans les principes des Rights of Man. Dans la deuxième lettre, Paine caractérise la pensée de Adams comme une " hérésie contradictoire », car il a prétendu défendre les constitutions américaines à partir de principes monarchiques qui en sont l'antithèse. L'apostasie de Adams est précoce. Paine explique qu'il a eu des doutes sur son républicanisme depuis 1776 et sa critique de Common Sense dans les Thoughts on Government. Adams critiquait en effet le célèbre pamphlet républicain de Paine et défendait le modèle anglais : «John était pour l'indépendance parce qu'il en attendait son élévation personnelle, mais il n'était pas difficile de comprendre [...] que sa tête était pleine de rois, de reines et de valets comme dans un vrai jeu de cartes $\rangle^{13}$. La rumeur publique disait que Adams n'était pas défavorable à une forme de gouvernement

(10) Letter I, p. 383.

(11) Letter VI, p. 409.

(12) Letter II, p. 385.

(13) Idem, p. 389. 
héréditaire et qu'il avait évoqué l'idée que, puisque Washington n'avait pas d'enfant, la présidence pourrait prendre une forme transmissible dans une branche collatérale de sa famille ${ }^{14}$. Racontar ou fait certain? Peu importe pour Paine qui y voit une confirmation des inclinations théoriques du deuxième président. Il est certain que Adams lui-même a donné prise aux critiques qui le présentaient comme un monarchiste déguisé. Les formes prises par ses tournées présidentielles, l'usage incongru pour les républicains de festivités organisées pour son anniversaire avait fait les choux gras de la presse républicaine aux États-Unis et même en France ${ }^{15}$. Une fois installé à la présidence par ses amis, écrit Paine, « ses principes (s'il en eut jamais) se corrompirent » et il devint un véritable « contre-révolutionnaire $»^{16}$.

L'apostasie des principes n'est pas une question individuelle mais collective. Paine revient sur cette question dans sa sixième Letter. Si l'Amérique a été plongée dans les affaires européennes, c'est parce que les administrations de Washington et de John Adams « ont apostasié les principes de la Révolution. Élevés à une hauteur à laquelle ils ne pouvaient prétendre, et pour laquelle ils n'avaient pas assez de jugement, ils se sont retrouvés comme des plumes flottant au vent au gré des moindres passions et de l'orgueil ${ }^{17}$. Que la faction ait été ou non traversée de divisions internes ne fait rien à l'affaire, car c'est justement la défense des intérêts privés contre la Res Publica qui est à l'origine de l'existence d'une faction ${ }^{18}$.

Troisième élément: la faction considère l'exercice du gouvernement, non comme une délégation de pouvoir et de souveraineté des citoyens mais comme une propriété privée, une Res privata, un monopole transmissible à une élite de gouvernants «naturels». Le peuple est aliéné comme peut l'être une propriété. Le seul véritable objectif de la faction est de détruire le gouvernement représentatif pour lui substituer une « monarchie » sans roi :

« Le système du gouvernement représentatif, l'orgueil et la gloire de l'Amérique, et le palladium de ses libertés, devait être renversé et aboli.

(14) Idem.

(15) Marc Belissa, «La République américaine vue par les républicains français sous le Directoire » dans Pierre SERNA (dir.), Républiques sœurs. Le Directoire et la Révolution atlantique, Rennes, PUR, 2009, p. 103-122.

(16) Letter VIII, édition Philip S. FonER, p. 954.

(17) Letter VI, p. 413.

(18) Letter VIII, p. 956. 
La prochaine génération ne devait pas être libre. Le fils devait ployer son cou sous le pied de son père, et vivre, privé de ses droits, sous la loi de l'hérédité $\gg{ }^{19}$.

L'idée de legs républicain et de l'enchaînement des générations est ici particulièrement présente. Imposer le modèle anglais et la corruption qui le fonde et changer le principe représentatif par un système où les dirigeants tiendraient leur pouvoir non plus de leurs mandants, mais d'une forme de «tenure » héréditaire, tel est l'objectif de la faction. Dans un texte politico-satirique de juin 1804 éreintant l'éloge funèbre d'Alexandre Hamilton (tué en duel par Aaron Burr) par son archi-ennemi Gouverneur Morris, Paine accuse Hamilton lui-même d'avoir «travaillé pour établir une constitution qui aurait privé les citoyens de toute sorte du droit de suffrage et se serait placé lui-même, [...], en possession d'un gouvernement à vie $»^{20}$.

Quels moyens la faction a-t-elle employés pour arriver à ses fins?

Elle a tout d'abord saturé l'espace public de mensonges et de détournement rhétoriques pour dissimuler ses objectifs.

Pour Paine, le rôle de la presse fédéraliste est essentiel, elle est en quelque sorte "la contre-institution » qui détourne les citoyens des principes et permet à la faction d'agir en dissimulant ses objectifs et en flattant les intérêts individuels. La presse fédéraliste "représente tout le peuple d'Amérique comme absolument dénué de principes publics et de mœurs privées $\rangle^{21}$. L'absence de principes républicains est la raison de sa « nullité », la presse fédéraliste n'est qu'un «néant bruyant ». Bien que puissante, la presse fédéraliste ne fait de mal qu'à ceux qui la lisent ${ }^{22}$. Les insultes contre Paine lui-même et Jefferson abaissent ceux qui les profèrent et il est inutile de répondre aux attaques personnelles. Il est à noter que Paine a toujours laissé dire et laissé imprimer à peu près toutes les calomnies et n'a jamais daigné répondre que dans ses propres écrits et de manière détournée aux attaques féroces dont il était la cible. Ce n'est que lorsque des membres des législatures d'État ou des officiers publics défendaient ces calomnies, comme dans le cas de Turner et Hulbert, deux représentants dans les législatures du Massachussetts et de

(19) Letter II, p. 388-389.

(20) Remarks on Gouverneur Morris's Funeral Oration, 7 août 1804, dans édition Philip S. FONER, tome II, p. 959.

(21) Letter I, p. 383.

(22) Letter IV, p. 399. 
Virginie en mars 1805 qui avaient attaqué Jefferson dans la presse, qu'il prend la plume pour y répondre ${ }^{23}$. Le langage de la presse fédéraliste est un reflet de l'absence de principes de la faction et donne à voir sa nullité, comme l'écrit longuement Paine dans sa dernière lettre aux citoyens américains ${ }^{24}$.

Mais sans le vouloir, la presse fédéraliste a fait le jeu des Républicains :

«Ces journaux ont été d'une grande aide à la cause républicaine, non seulement à la honte supplémentaire qu'ils ont apportée à leur propre faction honteuse, mais aussi en servant par contraste à mettre en valeur la décence et les arguments appuyés sur les bons principes des journaux Républicains $»^{25}$.

Cette remarque va plus loin que la simple polémique. Comme l'ont montré les historiens, l'une des causes de la défaite des fédéralistes est que leur conception de la High Politics excluant le peuple des débats pour la resserrer parmi l'élite sociale était contradictoire avec l'existence d'une presse à vocation populaire. Il est évident qu'en entrant dans le jeu du débat public large, y compris pour tenter de discréditer les Républicains, les Fédéralistes ouvraient sans le vouloir le champ démocratique. Paine en a conscience et il y voit une des raisons de la perfectibilité du régime républicain qui oblige même ses ennemis à se placer sur le terrain de l'extension de la publicité des principes.

Un autre des aspects de la dissimulation inhérente aux factions est le détournement des appellations politiques et en particulier de la première d'entre elles : les termes de "fédéralisme» ou de "Fédéralistes ». C'est, selon lui, à une véritable usurpation du vocabulaire que s'est livrée la faction. Ainsi dans la première Letter, il précise : «Je ne sais pas pourquoi on les appelle ainsi, car leurs principes sont antifédéralistes et despotiques ». Le mot «fédéralisme » n'a en lui-même aucun sens. On peut se fédérer pour perpétrer un crime ou accomplir une bonne action: "Ces hommes se sont-ils fédérés pour soutenir les libertés de leur patrie ou pour les renverser? Pour augmenter son renom ou pour se battre sur sa dépouille? $\gg^{26}$. En réalité, explique Paine, le seul

(23) To Mr. Hulbert of Sheffield..., 12 mars 1805, dans édition Philip S. FonER, p. 975-979 et Another Callender, Thomas Turner of Virginia, 23 juillet 1805, Idem, p. 980-988.

(24) Letter VIII, p. 951.

(25) Idem, p. 950.

(26) Letter II, p. 385 
sens positif du terme fédéraliste est à replacer dans un contexte qui est celui de la phase dite « critique » de la création de la République américaine dans les années qui suivent la victoire militaire. Paine rappelle que dans cette acception, la seule qui possède un réel sens politique, lui-même a été l'un des premiers "fédéralistes » puisqu'il a proposé très tôt, dit-il, la constitution d'une forme de gouvernement fédéral et républicain, à l'image de ce que proposait Mably dès $1784^{27}$. Le vrai fédéralisme était celui qui considérait que le lien républicain entre les États était trop lâche et que les lois d'un État interféraient avec celles des autres ${ }^{28}$. Paine rappelle au passage que les « anti-Fédéralistes » ne l'étaient pas tant que cela et que ce qui faisait problème dans le débat de ratification de la constitution en 1787 était non l'existence ou non d'une forme d'État fédéral mais des points constitutionnels comme la question de la forme et de l'exercice du pouvoir exécutif ou des milices, etc. Ce débat était tout à fait légitime, car, explique Paine : « Une République ne doit pas être seulement dans ses principes, mais aussi dans ses formes $»^{29}$. La méfiance vis-à-vis du pouvoir présidentiel n'était que trop justifiée estime Paine qui en profite pour égratigner Washington. Le terme de "fédéralisme » a donc pour la faction le rôle d'un écran de fumée destiné à masquer la nullité de ses principes et la destruction du véritable fédéralisme.

Dans un de ses derniers textes du 17 octobre 1806 intitulé $A$ Challenge to the Federalists to Declare their Principles, Paine répète le caractère fondamental des principes dans la définition des groupes politiques ${ }^{30}$. Les « anciens noms de Whigs et de Tories » ont été remplacés par ceux de « Républicains » et de « Fédéralistes ». Ces derniers sont les héritiers de ceux qui ont rejeté les principes fondateurs de la République. Les désignations de partis ne possèdent pas de contenu en eux-mêmes, ce sont leurs principes qui sont déterminants :

« Le mot de Républicain a en lui-même un sens, bien qu'il ne soit pas très positif, si ce n'est qu'il est opposé à la monarchie; mais le mot de Fédéraliste n'en a aucun. Ce n'est qu'un mot vide de sens. On peut l'utiliser pour un gang de voleurs fédérés pour commettre quelque crime, ou

(27) Gabriel Bonnot de MABLy, Observations sur le gouvernement et les lois des ÉtatsUnis d'Amérique, dans Euvres complètes, Paris, an VII, tome VIII.

(28) Letter II, p. 386.

(29) Idem, p. 388.

(30) A Challenge to the Federalists, édition Philip S. FonER, tome II, p. 1007-1010. 
pour n'importe quelle association. Quand les hommes forment des partis politiques, il est d'usage qu'ils fassent une déclaration de leurs principes, mais les Fédéralistes ne déclarent pas ce que sont leurs principes. Nous pouvons en déduire, soit qu'ils n'en ont aucun [...] soit qu'ils sont trop mauvais pour être déclarés ».

Les principes des Républicains sont déclarés : défense du gouvernement représentatif, de la paix entre les nations, refus de la guerre et d'une politique de puissance, rejet d'un système fisco-financier conçu pour enrichir une partie de la population au détriment des autres... Mais le principe fondateur est que la souveraineté « réside dans la grande masse du peuple ». Les Républicains ne reconnaissent donc aucune forme de gouvernement héréditaire ou d'aristocratie « naturelle».

Les « principes » des Fédéralistes étant nuls ou implicites, on doit les déduire de leurs actes, or ils se sont opposés aux suppressions des taxes proposées par Jefferson, ils ont voté pour la création d'une armée permanente et ont manipulé l'opinion pour la faire accepter et pour imposer un gouvernement à l'anglaise. Leurs « principes » sont donc ceux de la monarchie anglaise corrompue. Dans sa huitième Letter, Paine utilise des formules percutantes pour asséner l'idée de l'absence de principes républicains de la faction fédéraliste. Le fédéralisme sous sa forme partisane est un « indescriptible néant » :

« Demandez à un homme qui s'appelle lui-même un Fédéraliste de définir ce qu'est le fédéralisme, il ne le pourra pas. Demandez-lui quels sont ses principes et il n'en aura aucun à donner. Le fédéralisme est donc, dans le domaine du gouvernement, similaire à ce qu'est l'athéisme dans le domaine de la religion, un rien nominal sans principes $»^{31}$.

Les moyens de la propagande ne suffisent pas à asseoir le pouvoir de la faction et Paine insiste sur les projets militaires et fisco-financiers de Hamilton. On sait que Hamilton avait défendu l'idée d'une armée permanente et d'une marine de guerre susceptible de fonder une puissance militaire respectable. Pour les Républicains, ce projet est le pendant de ses trois rapports d'économie politique de 1790-1791 (Report on Public Credit, sur la Banque, puis sur les manufactures) visant à créer une sorte de complexe militaro-fisco-commercial et industriel à l'image de l'Angleterre. 
Comment faire accepter un tel plan? En instrumentalisant la peur et la religion. La peur de la France et de sa Révolution et la peur des ministres du culte qui s'enrôlèrent au service de la croisade contre la France « athée ». La Quasi-War avec la France entre 1795 et 1798 est donc pour Paine avant tout une manipulation destinée à liquider les principes républicains et à soumettre le peuple à une élite de négociants, de spéculateurs et d'administrateurs privés ou publics. Une armée permanente et donc de larges rentrées fiscales étant nécessaires pour asservir le peuple, on inventa des dangers imaginaires justifiant la croissance des effectifs militaires, alors qu'une analyse de la situation européenne aurait montré que la guerre franco-anglaise empêchait toute tentative d'invasion de l'Amérique par la France ou tout autre belligérant ${ }^{32}$.

Pourtant, écrit Paine, une partie des Américains y a cru et a soutenu cette politique absurde, mais la responsabilité de cette dérive n'est pas dans le peuple, dans la démocratie ou dans le gouvernement représentatif. Le peuple peut être trompé provisoirement, mais il n'est pas stupide et cet épisode ne saurait remettre en cause l'excellence de la République. Ce sont les mensonges de la faction qui portent la responsabilité de cet oubli momentané des principes.

La peur de l'invasion, des troubles internes justifient les Alien and Sedition Acts de 1798 et les procès qui en ont été la suite. Rappelons qu'en juin-juillet 1798, les Fédéralistes firent adopter une législation répressive allongeant notamment de 5 à 14 ans le délai de résidence pour obtenir la citoyenneté américaine. Il est vrai que de nombreux émigrés britanniques fuyant la répression s'étaient réfugiés aux États-Unis, or ces émigrés étaient pour la plupart de farouches républicains, influencés par les écrits de Paine. Le 14 juillet (on voit le symbolisme de la mesure), le Congrès adopta le Sedition Act par une majorité de 44 voix contre 41. Cette loi permettait de poursuivre, d'arrêter et de condamner à des amendes et à des peines d'emprisonnement toute personne convaincue d'avoir prononcé des discours ou écrits de manière «fausse, scandaleuse et malveillante » sur le gouvernement et ses officiers. Il s'agissait évidemment d'une loi dirigée contre la presse républicaine et contre les sociétés démocratiques-républicaines. Une quinzaine de journalistes furent poursuivis, dont dix furent condamnés à des amendes et emprisonnés pendant quelque temps. Plusieurs personnes furent poursuivies pour avoir pétitionné contre les lois répressives ou pour avoir érigé un arbre de la 
liberté ou même pour avoir dit que les États-Unis avaient une armée permanente. C'est contre ces lois répressives que les Kentucky and Virginia Resolutions furent adoptées à la fin de 1798 par les législatures de ces États ouvrant la voie à la contre-attaque des Républicains et à l'élection de Jefferson.

Paine qualifie ce moment de Reign of Terror à l'image de l'expression que les radicals anglais utilisaient pour qualifier la législation répressive anglaise à partir de 1793. Les biographes de Paine ont vu une exagération inutile dans l'utilisation de ce terme pour qualifier le régime des Alien and Sedition Acts ${ }^{33}$, mais les contemporains l'utilisaient largement et s'ils n'assimilaient pas exactement la «Terreur» française au Reign of Terror anglais et américain, son utilisation renvoyait à l'idée de législation d'exception dans une république et non à l'imagerie contrerévolutionnaire de la "Terreur». Paine s'interroge sur le succès temporaire de la stratégie de la faction. Comment expliquer le fait qu'une partie de l'opinion ait donné dans le panneau de la peur de la Révolution française et ait accepté des lois antidémocratiques? Il répond que le mensonge d'une faction peut tromper pendant un temps très court une majorité du peuple, mais que la vérité trouve toujours son chemin, car les mensonges s'ajoutant aux mensonges forment un amas inextricable qui finit par s'effondrer sur lui-même. Ainsi, la campagne anti-française de 1797-1798 faisant croire à la possibilité d'une invasion d'incendiaires ou même de troupes françaises était tellement ridicule qu'elle n'a pu jouer longtemps le rôle de justification de la politique fédéraliste ${ }^{34}$.

L'instrumentalisation de la religion est un autre des thèmes que l'on retrouve dans les écrits de Paine et notamment dans sa lettre du $1^{\mathrm{er}}$ janvier 1803 en réponse à Samuel Adams ${ }^{35}$. L'historiographie a, le plus souvent, utilisé la lettre qu'envoie le vieux révolutionnaire à Paine comme une preuve de l'isolement de l'auteur de The Age of Reason dans une Amérique religieuse et rejetant le déisme. Pourtant, l'examen des deux lettres montre que le problème en discussion n'est pas tant le rejet de la critique du christianisme de The Age of Reason mais les conséquences politiques d'une association du parti républicain avec les opinions religieuses de Paine dans le contexte des débuts de la présidence de Jefferson. Pour Paine, l'instrumentalisation de la religion par les ennemis des Républicains n'est qu'une forme de manipulation : «La Religion n'est pas la cause, mais le

(33) Voir notamment Jules Ayer, op. cit., p. 164-165.

(34) Letter III, p. 393.

(35) Letter to Samuel Adams, édition Eric FonER, p. 415-421. 
prétexte. Ils la mettent en avant pour se cacher derrière elle $»^{36}$. Pour réaliser leurs projets liberticides, les Fédéralistes ont enrôlé la chaire et les orateurs dans leur croisade antirépublicaine. Paine s'attaque particulièrement au prédicateur Nathaniel Emmons qui n'avait pas hésité à tonner en chaire contre le président élu et ses électeurs.

Paine revient sur la question dans sa Letter du 12 mars 1803. Il insiste sur la contradiction morale de l'association entre religion et guerre dans le discours de la faction qu'il oppose à l'association républicaine entre la paix et la morale ${ }^{37}$. Paine cible les discours bellicistes des Fédéralistes dans l'affaire de la Louisiane. En effet, les principaux leaders fédéralistes entonnaient le clairon guerrier pour obtenir une déclaration de guerre à la France ou du moins une occupation de la Nouvelle-Orléans qui aurait eu le même résultat, et une partie du clergé d'obédience fédéraliste demandait la guerre à longueur de sermons. Il en appelle au clergé pour que ses membres laissent de côté leurs arrière-pensées électoralistes et leurs sermons bellicistes et reconnaissent l'habilité et la moralité de la politique du gouvernement de Jefferson qui a réussi à préserver la paix. L'alliance entre les Fédéralistes et le clergé est, insiste-t-il, un bloc sans principes, ni morale ${ }^{38}$.

Pour Paine et les Républicains, le règne de la faction s'est terminé par son échec retentissant. La « Révolution de 1800 » a balayé tous les complots. Le peuple a cessé d'être la dupe des mensonges, il s'est ressaisi et a compris que la République et ses principes étaient en danger. Comment expliquer ce retournement? Comment assurer la défense de la République contre les factions? Comment élargir la démocratie américaine? Quels mécanismes constitutionnels mettre en place pour permettre à la perfectibilité du régime républicain de donner sa pleine mesure?

\section{Perfectibilité de la République}

Dès Common Sense et Rights of Man, Paine avait affirmé qu'une des raisons de la supériorité du régime représentatif et républicain démocratique sur tous les autres était qu'il était le seul à pouvoir s'autoréformer sans révolution, ni troubles par le jeu de l'expression de la souveraineté de tout le peuple. La République n'est pas un régime dans lequel le peuple

(36) Idem, p. 418.

(37) Letter VI, p. 409.

(38) Letter VIII, p. 949. 
ne peut pas se tromper ou être trompé, mais il est le seul dans lequel le peuple lui-même peut revenir sur ses erreurs et se perfectionner ${ }^{39}$. Cette « perfectibilité » des républiques est, selon Paine, la cause principale de l'échec fédéraliste. Mais, pour lui, la Révolution de 1800 n'est pas la fin du processus d'autoréforme. La République doit être refondée en permanence par la vigilance des citoyens et par l'exercice sans entraves de leurs droits. D'où la nécessité de réformer les constitutions d'État qui contenaient encore des limites au suffrage ou des dispositions favorisant les aristocraties "naturelles ». C'est le sens de son engagement auprès des réformateurs de Pennsylvanie au printemps de 1805, ou de ses propositions adressées à ceux du Connecticut l'année précédente et de ceux de l'État de New York en 1806.

La base de la perfectibilité de la République américaine réside avant tout dans la qualité du peuple américain, mais aussi dans le fait qu'il s'est emparé des principes des droits de l'homme pour fonder ses pratiques politiques. Ce que Paine appelle le Common Sense est l'esprit même de 1776, il est trop partagé selon lui pour qu'une faction puisse longtemps duper le peuple ${ }^{40}$. Une erreur commise par une génération peut être rattrapée par elle-même ou par la suivante. Là encore, il s'agit d'un thème essentiel chez Paine (sans doute hérité de Locke), une génération ne peut enchaîner celles qui lui succèdent, les pères ne peuvent asservir leurs enfants : le gouvernement américain étant fondé sur le système représentatif, une erreur peut être corrigée par le même processus rationnel par lequel la Constitution a été créée. Le peuple est en permanence « constituant».

Paine prend l'exemple des premières mesures prises par le gouvernement Jefferson, notamment avec l'abolition des lois scélérates (et le remboursement des amendes imposées aux journalistes!), la suppression d'une partie des taxes créées par les Fédéralistes et la diminution de la dette.

Les résultats des élections prouvent la perfectibilité du gouvernement représentatif et sa capacité d'éviter les affres des troubles civils :

«ÉLIRE et REJETER, est la prérogative d'un peuple libre. Aucune période n'a autant témoigné de l'excellence du système représentatif et sa supériorité sur tous les autres depuis l'acquisition de l'indépendance que le moment que nous vivons. Si l'Amérique avait dû subir la malé-

(39) John Keane, Tom Paine. A Political Life, Londres, Bloomsbury, 1995, p. 510.

(40) Letter I, p. 383. 
diction de la monarchie héréditaire de John Adams ou du Sénat à vie de Alexander Hamilton, nous aurions été obligés de chercher dans la guerre civile ce que nous avons obtenu par l'expression de la volonté publique. Un appel aux élections décide mieux qu'un appel aux armes $»^{41}$.

Mais un peuple vertueux et un régime représentatif ne sont rien sans un rappel des principes; c'est sur leurs principes ou leur absence de principes que les candidats et les partis doivent être jugés. Sans principes, l'élection et la représentation ne sont que des fictions ${ }^{42}$.

Paine applique ces maximes à des faits précis et il revient longuement sur les intrigues d'Aaron Burr au sein de son propre parti et avec certains Fédéralistes, pour être élu Président à la place de Jefferson. En effet, dans les premières élections américaines, le système du « ticket» avec un candidat à la présidence et un autre à la vice-présidence n'existait pas encore. Théoriquement, Burr ayant obtenu le même nombre de voix républicaines aurait pu réclamer le poste de Président contre Jefferson, devenant ainsi une sorte d'otage des Fédéralistes qui lui aurait apporté leurs voix pour empêcher Jefferson d'entrer en fonction. Paine met en garde contre tout type d'intrigues « politiciennes » qui ne visent qu'à empêcher la volonté populaire de s'exercer. De même, Paine prend position pour la mise sur pied d'une commission d'enquête sur la conduite de l'administration Adams. Il précise qu'il ne s'agit pas d'une vengeance ou d'une volonté de « réaction » (au sens français de l'époque), mais de la nécessité de mettre en pratique la perfectibilité du régime républicain. La faction fédéraliste a gouverné dans l'opacité et le secret, ne donnant jamais les informations sur le danger d'invasion qui justifiait sa politique répressive, il est donc indispensable de faire la lumière et de rendre publique la politique du gouvernement précédent. Paine défend donc une forme de « censure » du gouvernement qui permettrait de comprendre les erreurs commises et d'en empêcher le retour. La réconciliation des partis ne peut se faire sur la base de l'oubli, mais sur celle de la publicité des principes et des actes devant 1 'opinion de tous les citoyens ${ }^{43}$.

Une fois la réélection de Jefferson assurée en 1805, William Duane et John Binns, deux dirigeants républicains de Pennsylvanie s'adressèrent à Paine pour obtenir son soutien dans leur campagne pour la révision de la constitution d'État de 1790, qui avait été elle-même le fruit d'une

(41) Letter III, p. 392.

(42) Letter V, p. 405.

(43) Letter VI, p. 414. 
révision de la constitution originelle de 1776. Les réformateurs de l'État de New York cherchaient à faire modifier un certain nombre de dispositions constitutionnelles, notamment celles concernant l'octroi de chartes privées par le pouvoir exécutif. Paine publie ses contributions dans l'Aurora et en brochures séparées. Il s'agit de deux textes intitulés Constitutions, Governments, and Charters du 21 juin 1805 et de To the Citizens of Pennsylvania on the Proposal For Calling a Convention d'août de la même année ${ }^{44}$. Dans ces deux textes, Paine pose une série de questions constitutionnelles qui ont toutes pour but d'élargir les formes et la nature de la démocratie et de créer des mécanismes permettant à la perfectibilité républicaine de s'exprimer.

Pour Paine et une grande partie des Républicains de Pennsylvanie, la constitution révisée de 1790 a été un recul par rapport à celle, beaucoup plus démocratique, de 1776. La première critique qui lui est adressée est qu'elle fait la part trop belle aux avocats, à la chicane et aux officiers de justice contre les citoyens. La réflexion de Paine sur les complications de la justice s'insère dans un débat plus général consécutif à l'adoption des Alien et Sedition Acts et surtout du Judiciary Act que les Fédéralistes imposèrent dans l'entre-deux entre leur défaite électorale et leur sortie de l'administration présidentielle. Les Fédéralistes avaient appointé seize nouveaux juges fédéraux itinérants, tous des Fédéralistes qui avaient soutenu les lois répressives sans état d'âme. Indépendamment de cette affaire, l'idéologie républicaine était hostile à un régime de justice complexe où les avocats s'enrichissaient des difficultés de la procédure, d'où une volonté de simplifier la justice et d'obtenir l'abrogation du Judiciary Act. À la suite d'une bataille juridico-constitutionnelle connue sous le nom d'affaire Marbury, la Cour suprême (à majorité fédéraliste) réussit néanmoins à renforcer considérablement son pouvoir, inaugurant une tradition américaine dans laquelle le pouvoir judiciaire suprême affirmait son autorité sur les décisions du pouvoir législatif, à la grande fureur des Républicains qui y voyaient une infraction au principe lockéen de la subordination de tous les pouvoirs au pouvoir législatif, suprême car expression de la volonté générale.

Paine rappelle le contexte dans lequel les premières constitutions d'État furent adoptées : celui de la guerre d'indépendance et donc d'une urgence qui permit au modèle anglais alors encore présent dans tous les

(44) Constitutions, governments and charters, 21 juin 1805, édition Daniel ConwAY, tome IV, p. 467-469. To the Citizens of Pennsylvania, août 1805, Idem, p. 457-466. 
esprits de s'insinuer dans une partie des dispositions constitutionnelles, introduisant les principes de la corruption de l'Ancien Monde dans le Nouveau. Par ailleurs, les constituants manquaient d'expérience et il n'est pas étonnant que les textes aient nécessité des ajustements et des modifications. Parmi les faux principes qui se sont alors imposés figure, selon Paine, celui de la concurrence des pouvoirs dans lequel l'exécutif, le judiciaire et le législatif sont imbriqués. Paine visait notamment les dispositions qui donnaient à certains exécutifs d'État un droit sinon de veto, du moins de ratification de certains actes législatifs. Même si ce pouvoir était exercé collégialement comme dans l'État de New York (par un Conseil de révision composé du gouverneur, du chancelier et des juges), il était néanmoins dangereux, car il créait de la confusion et affaiblissait le pouvoir législatif en légitimant l'intervention du pouvoir exécutif et même du pouvoir judiciaire qui n'était, selon Paine, qu'une branche particulière de l'exécutif.

Pour justifier la révision constitutionnelle, Paine revient aux principes du régime représentatif en rappelant la définition du pouvoir constituant lockéen :

«Une constitution est un acte souverain du peuple. Le gouvernement est une créature de la constitution, il est produit par elle et lui doit son existence. Une constitution définit et limite les pouvoirs du gouvernement qu'elle crée. Il s'ensuit naturellement et logiquement que l'exercice gouvernemental de quelque pouvoir qui n'est pas autorisé par la constitution est un pouvoir supposé et donc illégal $»^{45}$.

Paine prend ici position en faveur de ce que les contemporains appelaient la strict construction, c'est-à-dire d'une conception limitative de la prérogative gouvernementale, contre les Fédéralistes qui, eux, défendaient une broad construction à l'anglaise qui donnait à l'exécutif une large prérogative. Il applique la strict construction au problème des chartes et des monopoles accordés par les exécutifs d'État à des entreprises, souvent bancaires. Pour Paine, le fait d'accorder des chartes créant des obligations contractuelles pluriannuelles entre ces entreprises privées et l'ensemble des citoyens contrevenait à la perfectibilité républicaine. Alors que les élections étaient annuelles, les chartes ne l'étaient pas, les électeurs étaient donc enchaînés par les choix effectués antérieurement. Le pouvoir de «censure » des actes du législatif et de l'exécutif des citoyens était

(45) Constitution, governments..., op. cit., p. 467-468. 
alors réduit à néant. Pour éviter les engagements à long terme sur lesquels il serait impossible de revenir, Paine propose une forme d'engagement en deux temps : une législature pourrait présenter un bill établissant une forme de charte, mais ce n'est que la suivante qui pourrait lui donner effet, permettant ainsi la censure des citoyens par le biais des élections entre les deux passages législatifs. Paine propose que ce mécanisme de correction annuel soit adopté sous la forme d'un article constitutionnel, créant ainsi un droit des citoyens à censurer par les élections tous les actes du législateur, chartes et monopoles compris. Paine visait en particulier l'affaire récente de la Merchant's Bank de New York établie par la législature d'État et dans laquelle il voyait le réservoir d'une corruption à l'anglaise.

Dans le cas de la Pennsylvanie, le problème était plus large, les réformateurs cherchant à obtenir la convocation d'une Convention d'État afin de faire abroger toutes les dispositions limitatives de la démocratie adoptées en 1790, notamment celles sur le droit de suffrage. Le reproche qui a justifié la révision était qu'elle ne prenait pas assez de précautions contre la " précipitation » et les décisions prises dans «l'enthousiasme », ce qui dans le langage politique des années 1790 visait directement le caractère supposé trop populaire et démocratique de la constitution originelle. La révision avait introduit des formes plus lentes, et surtout, selon Paine, influencée par la pratique parlementaire anglaise, elle avait créé une forme de veto exécutif sur le législatif. Paine reprend le thème radical du Norman Yoke (le joug normand) pour montrer que toute la tradition parlementaire anglaise de la prérogative exécutive découle non des principes, mais de la conquête et de la violence. La conclusion découle :

«Ce pouvoir négatif dans les mains d'un seul individu devrait être aboli constitutionnellement. C'est un pouvoir dangereux. Il n'y a pas de règles qui en fixent l'usage. Il est discrétionnaire et arbitraire et la volonté et le caractère de la personne qui le possède est la seule règle $»^{46}$.

La deuxième disposition visée par Paine est l'organisation de la justice. Il se prononce pour le développement des pratiques d'arbitrage dans toutes les affaires civiles pour limiter le pouvoir des juges et des avocats dont la pratique est d'obscurcir ce qui est clair par des procédures qui n'existent que pour leur fournir un revenu. Là encore, Paine propose de faire de l'arbitrage un principe constitutionnel pour en finir avec la

(46) To the Citizens of Pennsylvania, op. cit., p. 457. 
«prostitution » des causes par les avocats qui ne vivent que des injustices qu'ils prétendent redresser ${ }^{47}$. Les formes de la justice en Amérique sont encore trop « anglaises ». Paine propose d'en finir avec elles, avec la jurisprudence coloniale, avec le langage même de la justice anglaise, truffé de latin et de "français judiciaire », incompréhensible pour tous, sauf pour les avocats qui en profitent pour établir leur monopole.

Le développement de l'arbitrage permettrait en outre de limiter le « patronage » qui favorise la corruption. En effet, Paine critique vivement le droit de patronage de l'exécutif lui permettant de placer ses « créatures » à des postes lucratifs. Autre critique : la durée excessive du sénat d'État (quatre ans) qui ne reflète pas les évolutions démocratiques de la législature élue annuellement. La différence de durée de la mandature remet en cause le principe de l'annualité des élections et donc les possibilités de réformes et par conséquent le principe de perfectibilité. Un Sénat de quatre ans n'est rien d'autre qu'une Chambre des Lords en miniature, une aristocratie élective, pâle copie de la constitution anglaise.

Enfin, Paine s'attaque aux restrictions censitaires. La constitution de 1776 était démocratique, mais dans la précipitation, elle n'a pas mis en place les mécanismes de correction et de vérification populaire des lois, c'est ce à quoi il faut remédier, mais il faut d'abord réaffirmer le droit fondamental d'élection pour tous :

« La constitution de 1776 était conforme à la Déclaration d'Indépendance et à la Déclaration des Droits, ce que la présente constitution n'est pas, car elle établit des distinctions artificielles entre les hommes du point de vue du droit de suffrage [...]. Nous voyons tous les jours des riches devenir pauvres et ceux qui étaient pauvres s'enrichir. La richesse n'ayant aucun caractère de stabilité ne peut et ne doit pas être un critère de droit. L'homme est homme dans toutes les conditions... ${ }^{48} \gg$.

Le droit de suffrage est donc un droit naturel qui ne peut et ne doit pas être enfreint par des lois constitutionnelles.

L'Amérique doit donner une fois de plus l'exemple au vieux monde que le régime représentatif républicain et démocratique est supérieur à tous les autres. C'est en lui permettant d'exercer sa qualité première, la perfectibilité, que les principes qui le fondent pourront devenir ceux de l'humanité tout entière :

(47) Idem, p. 458.

(48) Idem, p. 462. 
«Les gouvernements des autres nations, qui se proclament vainement civilisées, ont été établis dans le sang. Pas une seule goutte de sang n'a été versée aux États-Unis dans le processus de formation des constitutions et des gouvernements par son propre système pacifique. Le vote, ou un simple "oui" ou "non", sont plus puissants que les baïonnettes... »"

La question des mécanismes de correction démocratique continue d'occuper Paine jusque dans ses derniers écrits et on le voit encore en 1807, alors que sa santé s'est dégradée, écrire au sénateur Mitchell de New York pour lui soumettre un amendement autorisant la destitution d'un juge de la Cour suprême par le Président sur la réquisition de la majorité des deux chambres afin d'éviter un pouvoir excessif et sans censure populaire du pouvoir judiciaire suprême ${ }^{50}$.

\section{La mémoire radicale de 1776}

Les textes des dernières années de Paine s'intéressent à la question de la "mémoire » de la Révolution américaine en fustigeant l'amnésie sélective des Fédéralistes. La légende grise en a fait un indice de son « décalage » par rapport aux enjeux de son époque, comme si le vieillard s'attaquait à la génération suivante dans un esprit de dénigrement. Ce n'est pas le cas. Les années 1800 sont bien celles du passage d'une génération à une autre. Les révolutionnaires de 1776 avaient déjà réfléchi au problème de la transmission des principes et des valeurs du républicanisme. On sait que les différentes traditions républicaines décrites par Pocock ou Skinner ou plus récemment dans le colloque du séminaire L'esprit des Lumières et de la Révolution ${ }^{51}$, insistent toujours sur la nécessité de régénérer l'esprit républicain à intervalles réguliers et de rappeler dans des célébrations et des « institutions » (au sens défini par Rousseau ou SaintJust) les principes qui fondent la Res Publica. Ainsi, Mably conseillait en 1784 aux Américains de réfléchir à un système de fêtes nationales pour rappeler aux générations futures la lutte pour la liberté et cimenter l'union. Benjamin Franklin avait posé la même question peu avant sa mort $^{52}$ : l'Amérique avait besoin d'une réitération de ses valeurs fondatrices et d'un encouragement à la vertu républicaine. D'où l'insistance

(49) Idem., p. 465.

(50) Moncure D. Conway, op. cit., p. 357.

(51) Marc Belissa, Yannick Bosc, Florence Gauthier (dir.), Républicanismes et droit naturel. Des humanistes aux révolutions des Droits de l'homme et du citoyen, Paris, Kimé, 2009.

(52) Craig Nelson, op. cit., p. 308. 
de Paine sur le lien à établir entre les principes de 1776 et ceux de 1800 contre l'absence de principes de la « faction » fédéraliste. Il fallait renouer le fil entre les générations et clore le moment fédéraliste de 1789-1800 en rappelant les valeurs révolutionnaires contre les interprétations «élitistes » et « exceptionnalistes » de la Révolution des Fédéralistes. Paine n'est donc pas confit dans le rappel de ses mérites passés et de ceux de sa génération, il est même en avance par rapport aux réflexions que les hommes de 1776 couchent sur le papier dans les années 1810-1820 $0^{53}$.

La question de la mémoire révolutionnaire apparait donc en filigrane dans la plupart des textes tardifs de Paine. Quand il rappelle dans la première Letter son rôle dans la phase initiale de la Révolution, il ne s'agit pas seulement d'un plaidoyer pro domo, mais aussi de l'affirmation de la légitimité de sa position d'écrivain patriote dans le débat public et de la continuité des principes de 76 et de ceux de la "révolution» de 1800 par-dessus ce qu'il estime être un entre-deux dans lequel ces principes ont été occultés. Il rappelle ainsi qu'à peine deux ans après son départ d'Amérique ont surgi les symptômes d'un oubli des principes alors même que ceux de la liberté se frayaient un chemin en Europe ${ }^{54}$. L'Amérique tourna alors, écrit Paine, le dos à sa propre gloire et rétrograda vers l'oubli des principes. Mais les vrais républicains maintinrent la flamme de la liberté. Paine utilise à plusieurs reprises l'image de «l'étincelle de l'autel de 1776 » dans ses textes tardifs, il en signe certains par ce pseudonyme. Cette étincelle de l'esprit de 76, inextinguible pendant ce qu'il appelle la « longue nuit de l'erreur », resurgit en 1800 dans toute l'Union, elle est la preuve du maintien de l'esprit de la liberté et de la raison. Paine se place dans la position de l'écrivain patriote (telle que l'a analysée Larkin $^{55}$ ) dont le rôle est, entre autres, celui d'un intermédiaire entre les générations, il est celui qui transmet le message de 76, inchangé depuis Common Sense et Rights of Man. Indépendant des partis, car ne recherchant aucune place, ni aucun office, se moquant des attaques fédéralistes, Paine se place sur le terrain de la citoyenneté républicaine et de la transmission civique.

(53) Idem, p. 337. Jefferson écrit à la fin de sa vie : «Tous, tous morts, et nous nous trouvons seuls au milieu d'une nouvelle génération que nous ne connaissons pas et qui ne nous connait pas [...] Je regrette de mourir maintenant dans la croyance que l'inutile sacrifice de la génération de 1776 soit jeté au loin par les folles et indignes passions de ses fils, et ma seule consolation est que je ne vivrais pas pour le pleurer $[\ldots] »$.

(54) Letter I, p. 382.

(55) Edward LARkin, Thomas Paine and the Literature of Revolution, New York, Cambridge University Press, 2005. 
Revenu après une longue absence, il peut donc jouer le rôle d'un témoin entre l'avant et l'après, entre 1776 et 1800 . Que constate-t-il? Tout d'abord, qu'une grande partie de ses amis n'a pas varié dans ses principes, ce sont évidemment les Républicains, mais une partie de ses concitoyens a usurpé le nom de Fédéralistes pour légitimer la création d'une faction anglophile, monocrate et belliciste. Les « vrais » Fédéralistes sont les Républicains, mais le fait doit être rappelé, car des milliers d'Américains de 1802 n'étaient pas adultes en 1787. Il faut donc dévoiler l'usurpation du terme pour qu'ils puissent se ressourcer aux vrais principes.

Contre les Fédéralistes qui lui font un crime d'avoir attaqué la figure de Washington dans sa fameuse lettre au président décédé ${ }^{56}$, il rappelle que ce n'est pas le combattant de la Révolution qu'il a attaqué, mais le président otage de la « faction » et tellement imbu de sa fonction qu'il a fait dériver la pratique exécutive dans un sens «monarchique ». Contre l'espèce de « culte de la personnalité » que les Fédéralistes ont essayé de faire jouer en " récupérant» la figure de Washington à leur profit, Paine rappelle que les généraux, comme les écrivains patriotes, et comme les simples miliciens, ont joué des rôles d'importance égale dans la Révolution qui n'est pas le fruit de l'action des grands hommes et des élites, mais celui de la conjonction de l'esprit républicain et du peuple ${ }^{57}$.

Ce qui est en jeu dans la polémique et dans ces rappels historiques n'est pas pour Paine une question de personnes, mais bien la question de la nature de la Révolution américaine. Ce travail de mémoire se retrouve notamment dans les textes de 1805-1806 contre Hulbert et Turner, deux fédéralistes qui s'étaient attaqués à Jefferson. Paine les signe de son deuxième pseudonyme transparent : A Spark from the Altar of 76 (le premier étant Common Sense). Révolution des principes, révolution populaire, radicale et démocratique comme le défendent les Républicains de 1800 ? Ou bien continuité avec la Glorious Revolution de 1688, défense paradoxale des libertés anglaises sous la direction des élites " naturelles » ne remettant pas en cause les hiérarchies existantes? C'est tout le problème de la radicalité ou de la modération, de l'exceptionnalisme et de l'universalisme. Contre les Fédéralistes qui commencent à défendre, par exemple dans la presse ou les sermons de Nathaniel Emmons ou de Noah

(56) Letter to George Washington, 30 juillet 1796, édition Daniel ConwAy, tome III, p. $213-252$

(57) Another Callender - Thomas Turner of Virginia, 23 juillet 1805, dans édition Philip S. FONER, tome II, p. 980-988. 
Webster ${ }^{58}$, l'idée d'une révolution américaine sage, non-violente, exceptionnelle et inscrite dans l'histoire des libertés anglaises, Paine reprend le flambeau des principes de 1776, ceux de l'universalisme de la Révolution américaine, matrice des révolutions qui l'ont suivie, ceux de la radicalité et de la démocratie contre le pouvoir des élites. Sa défense du rôle de l'écrivain patriote et de sa méthode de publicité « directe » sans référence à la culture des élites participe de cette défense de la démocratie radicale $^{59}$. La liberté étant opprimée en Amérique même, et la trahison tramée dans le gouvernement, il était impatient de pouvoir reprendre le flambeau de 76 :

« J'ai appris que mes amis étaient opprimés et j'avais hâte de prendre ma place parmi eux, et si d'autres temps qui mettent les âmes à l'épreuve [times to try men's souls] arrivaient, je pourrais en supporter avec eux la charge $»^{60}$.

L'expression « times to try men's souls », passée en quasi-proverbe, était tirée du pamphlet American Crisis écrit pendant la guerre d'Indépendance et qui, avec Common Sense, avait établi la réputation de Paine comme écrivain. La formule fit florès et en vint à symboliser l'énergie républicaine dans le danger. Paine établit donc un parallèle entre le combat de 1776 et celui de 1800 . Il s'agit de retrouver la radicalité républicaine de l'expérience américaine contre ce qui l'a dénaturé et tenté de la détruire depuis 1789. Dans la sixième Letter, il en appelle même à la mobilisation populaire autour de la mémoire radicale de la Révolution ${ }^{61}$.

Dès sa Lettre à l'abbé Raynal en 1782, Paine avait défendu l'idée que l'originalité de l'expérience américaine était précisément son universalité et son impact sur les relations entre les peuples, il persiste en 1803 en insistant sur le devoir de l'Amérique vis-à-vis de l'humanité. Si l'Amérique, au lieu de donner l'exemple d'une conduite morale entre les nations, se comportait avec elles comme des conquérants, elle ferait reculer la civilisation et son indépendance au lieu d'être un honneur universel deviendrait une malédiction pour elle-même et pour l'humanité tout entière ${ }^{62}$.

(58) Voir l'anthologie publiée par la fondation Ollin, SANDOz Ellis (ed.), Political sermons of the American founding era : 1730-1805, Indianapolis, Liberty press, 1990.

(59) Letter IV, p. 402.

(60) Idem.

(61) Letter VI, p. 415.

(62) Idem, p. 410. 
Le 7 juin 1805, il revient encore dans son ultime Letter sur le thème de l'opposition entre conception exceptionnaliste et universaliste de l'expérience révolutionnaire :

« L'indépendance de l'Amérique n'aurait pas ajouté grand-chose à son propre bonheur et absolument rien au monde si son gouvernement avait été formé sur les principes corrompus de l'ancien monde. C'est l'occasion de renouveler le monde et de mettre en avant un système de gouvernement nouveau dans lequel les droits des hommes seraient préservés qui a donné sa valeur à l'indépendance. [...] La seule indépendance aurait peutêtre pu être conquise par les armes et sans principes, mais alors jamais un système juste de gouvernement n'aurait vu le jour. En bref, ce furent les principes qui produisirent alors l'indépendance, car tant que les principes ne s'étaient pas diffusés dans tout le peuple, personne ne pensait même à l'indépendance $»^{63}$.

L'expérience américaine ne se réduit pas à l'indépendance, c'est bien la construction républicaine fondée sur le droit des hommes et les principes de la liberté universelle qui en est le fruit, et non la séparation des colonies de la métropole anglaise.

Paine ne se contente pas de rappeler ces principes, il les applique aux cas qui lui sont soumis. Ainsi dans les textes écrits au sujet des projets de réformes constitutionnelles en Pennsylvanie, il part également de sa position d'écrivain patriote détenteur de la mémoire radicale pour défendre l'extension du droit de vote à tous et briser tout ce qui dans les constitutions d'État limite encore la démocratie, d'où son combat sur le terrain constitutionnel. On remarquera la continuité de Paine sur ce sujet, lui qui s'était élevé contre la dérive censitaire de la Constitution de l'an III comme l'a étudié Yannick Bosc ${ }^{64}$.

De même, l'affaire provoquée par le refus d'un certain Ward, fédéraliste à New Rochelle, de permettre à Paine de voter à la fin de 1806 sous le prétexte qu'il était anglais et non-américain, n'est-elle pas pour Paine une question personnelle, mais une question de principe qui met en cause

(63) Letter VIII, p. 956.

(64) Yannick Bosc, «Thomas Paine et les Constitutions de 1793 et 1795 : critique de la république formelle » dans Thomas Paine ou la République sans frontières, Bernard VINCENT (dir.), Nancy, PUN, 1993; "Ordre social et révolution. Boissy d'Anglas et le rejet de la Déclaration de 1793 en l'an III », dans Roger Bourderon (dir.), L'an I et l'apprentissage de la démocratie, Éd. PSD Saint-Denis, 1995 ; La terreur des droits de l'homme et du citoyen, Paris, Publications de la Sorbonne, à paraître. 
la mémoire révolutionnaire. C'est ce qu'il écrit dans ses dernières lettres à Madison et Clinton en mai $1807^{65}$. Lui refuser le droit de vote n'était pas seulement une vengeance des Fédéralistes contre un de leurs ennemis, c'était aussi une manière de "déradicaliser » la Révolution et de faire disparaitre sa dimension universelle en niant la participation de l'homme qui symbolisait plus que tout autre les trois Révolutions d'Amérique, d'Angleterre et de France.

Les derniers textes de Paine posent des questions fondamentales pour l'évolution du républicanisme en Amérique. Celle de la place des partis est l'une des plus intéressantes. La République n'est pas une forme de gouvernement creuse. Sans les principes déclarés des droits de l'homme, sans les formes et les pratiques de la démocratie radicale, sans la possibilité pour le peuple de pouvoir censurer les actes des gouvernements, la République est une coquille vide, un régime nul dans lequel les factions prospèrent. Paine ne rejette pas la possibilité de « partis » qui défendraient des opinions divergentes - comme une partie de la tradition républicaine qui y voit le début de la décadence - mais ces partis ne sont légitimes que s'ils sont créés sur la base des principes de la démocratie radicale et des droits de l'homme, tout autre groupement ne peut être qu'une faction. L'apparition de factions dans une république est un phénomène préoccupant mais il n'est pas anormal, car la perfectibilité du régime représentatif républicain lui permet de les dévoiler et de les empêcher de nuire au système républicain lui-même. L'extension de la démocratie représentative par le biais du suffrage universel, du droit de censure du peuple et son activité constituante permanente est la garantie de la possibilité d'une amélioration constante du régime républicain. Le rôle de la presse et des écrivains patriotes est fondamental. Ils jouent le rôle d'une " institution » républicaine de contrôle des gouvernements et de leurs actes. Enfin, les derniers textes de Paine sont emblématiques d'un «moment», celui du passage de la génération révolutionnaire à la suivante. La République doit être perpétuellement refondée dans des « institutions » qui rappellent au corps civique l'origine de ses droits et la manière de les conserver. La dimension universaliste, cosmopolitique et radicale de la Révolution amé-

(65) Lettres à Jefferson, à Madison (3 mai 1807) à Clinton (4 mai), dans édition Philip S. FONER, tome II, p. 1486-1487. 
ricaine est une expérience républicaine offerte à tous les peuples contre les monarchies ou les régimes « monocratiques », contre les aristocraties « naturelles » ou celle de la richesse, contre la confiscation de la souveraineté de tout le peuple par des factions politiciennes.

Marc BeLISSA Université Paris-Ouest Nanterre la Défense 200 av. de la République 92000 Nanterre mbelissa@club-internet.fr 